\title{
The impact of COVID-19 pandemic on the dietary patterns and eating behaviour in Saudi adults
}

\author{
N.M. Al-Mana ${ }^{1}$, H.A. Awney ${ }^{1}$, T.A. Zareef ${ }^{2}$, F.A. Albathi ${ }^{1}$, F.A. Baeshen ${ }^{1}$, S.A. AlZahrani ${ }^{1}$ \\ and R. Abdullah ${ }^{1}$ \\ ${ }^{1}$ College of Applied Medical Sciences, Department of Clinical Nutrition, King Saud University for Health Sciences, \\ Jeddah, Saudi Arabia and \\ ${ }^{2}$ Saudi Electronic University, College of Health Sciences, Public Health Department, Jeddah, Saudi Arabia
}

The continuing spread of the coronavirus prompted countries to take a range of precautionary steps, starting with implementing a quarantine $^{(1)}$. As a result, these precautionary measures have affected the dietary patterns of the general population ${ }^{(1,2)}$. Therefore, this study aimed to examine the effect of quarantine during the COVID-19 pandemic on dietary patterns and eating behaviours in adults living in Saudi Arabia.

A cross-sectional study was carried out on a sample of Saudi adults living in Jeddah aged $\geq 18$ from both genders. A web-based online survey was used, and 258 adults living in Jeddah completed the survey. The questionnaire contained demographic data and dietary patterns information to assess changes in eating habits before and during the COVID-19 quarantine. Data were analyzed using IBM SPSS Statistics for Windows, version 26 (IBM Corp., Armonk, N.Y., USA).

During COVID-19 lockdown, $74 \%$ of the respondents reported eating lunch as their main meal of the day compared to $86 \%$ before $(p<0.001)$. Interestingly, more individuals reported dinner as their main meal after the lockdown of COVID-19 relative to before (before $=79.1 \%$, after $=84.1 \%, p=0.079$ ).

We found a significant difference in the number of snacks consumed during the lockdown $(\mathrm{M}=3.03, \mathrm{SD}=1.235)$ compared to before the lockdown $(\mathrm{M}=2.46, \mathrm{SD}=1.048),(p<0.001)$. Also, less fast food was consumed (before: $\mathrm{M}=2.30, \mathrm{SD}=0.648$, after: $\mathrm{M}=1.92, \mathrm{SD}=0.797, p<0.01$ ), with a tendency to eat more dessert during the lockdown period (before $=45 \%$, after $=58 \%, p<$ $0.01)$. Our data indicated an increased coffee and water consumption during the COVID-19 time [coffee: $(\mathrm{M}=1.86, \mathrm{SD}=0.657)$ compared to before the lockdown $(\mathrm{M}=1.78, \mathrm{SD}=0.566), p<0.01$; water: $(\mathrm{M}=1.82, \mathrm{SD}=0.673)$ compared to before $(\mathrm{M}=1.67, \mathrm{SD}=$ $0.680), p<0.001)$.

These results suggest that the COVID-19 pandemic resulted in dietary patterns changes among the population living in Jeddah. Our data show shifting in dietary and meal patterns included type and frequency of snack consumption and the main meal-type, with a significant increase in fluids consumption. Further investigation in other age groups and regions would be required.

\section{Acknowledgments}

I would like to Acknowledge all the participants in this study for their time and patience. I would like to express my acknowledgement to the research team in the Clinical Nutrition Department at King Saud bin Abdulaziz University for Health Sciences.

\section{References}

1. Di Renzo L, Gualtieri P \& Pivari F (2020) J Transl Med 18(1), 229.

2. Sidor A \& Rzymski P (2020) Nutrients 12(6), 1657. 